Article

\title{
Expression and Distribution of the Auxin Response Factors in Sorghum bicolor During Development and Temperature Stress
}

\author{
Dan Chen, Weian Wang, Yaqin Wu, Hui Xie $\odot$, Linfei Zhao, Qi Zeng * and Yonghua Zhan * \\ School of Life Science and Technology, Xidian University, Xi'an 710126, China; dchen@xidian.edu.cn (D.C.); \\ weian02.wang@gmail.com (W.W.); wuyq@ascend-bio.com (Y.W.); hxie@xidian.edu.cn (H.X.); \\ linfeizhao1@gmail.com (L.Z.) \\ * Correspondence: qizeng@xidian.edu.cn (Q.Z.); yhzhan@xidian.edu.cn (Y.Z.)
}

Received: 7 September 2019; Accepted: 25 September 2019; Published: 27 September 2019

\begin{abstract}
Auxin response factor (ARF) is a transcription factor that can specifically bind to the promoter of auxin-responsive genes in plants and plays an important regulatory role in plant growth and development. The previous studies have predicted 25 ARF genes in Sorghum bicolor (SbARFs) and indicated that $\mathrm{SbARFs}$ play complex roles in salt and drought stresses. In this study, we reclassified and analyzed the structures of ARFs in three plants, including sorghum, rice, and Arabidopsis. Phylogenetic analyses categorized 73 ARF into five classes. By studying the characterization of the structures, it was found that SbARFs from the same evolutionary branches showed similar motif patterns. Furthermore, the expression patterns of $S b A R F$ genes during development and temperature stress were investigated in sorghum. Quantitative transcription-quantitative polymerase chain reaction (qRT-PCR) results suggested that they had different expression patterns in vegetative and reproductive organs at various developmental stages. High and low-temperature treatments and qRT-PCR demonstrated some of them changed dramatically along with the increase of treatment time. Additionally, in situ hybridization results displayed that $S b A R F$ genes were accumulated in vascular tissues under temperature stress. These findings provide evidence that SbARFs may play important roles in sorghum vegetative development, reproductive development, and auxin response to temperature stress.
\end{abstract}

Keywords: ARF; Sorghum bicolor; expression patterns; development; high and low-temperature stresses

\section{Introduction}

Auxin is known to be involved in the regulation of growth and development and abiotic stress response. As an important family of proteins in auxin-mediated response, ARF can recognize the auxin-response elements (AuxREs) in the promoters of auxin-responsive genes and then activate or repress their expression [1,2]. Most ARF proteins consist of three parts: An N-terminal B3-type DNA binding domain (DBD) that may recognize the AuxREs, a variable middle region that may play as an activation domain (AD) or repression domain (RD), and two C-terminal domains (CTD: domain $\mathrm{III} / \mathrm{IV}$ ). The CTD is involved in protein-protein interactions by dimerizing with auxin/indole-3-acetic acid (Aux/IAA) family genes or even dimerizing between ARFs [1,3,4].

According to the characteristic of molecular structures, ARFs have been identified from different plant species, and their roles in auxin-related plant growth and development have been studied intensively. The first ARFwas identified from Arabidopsis (AtARF1) and, subsequently, 23 ARF members were found in Arabidopsis [5]. Since then, based on the genome-wide analysis, ARF gene family has been identified in more than 20 species of higher plants, such as 25 ARFs from rice [6], 36 ARFs from 
maize [7], 24 ARFs from chickpea [8], 20 ARFs from Tartary buckwheat [9], 39 ARFs from litchi [10], and other species. The gain-of-function and loss-of-function mutants help a thorough understanding of the crucial role of ARFs. By using Arabidopsis mutants, many studies have been done about it in recent years. As transcriptional repressors, AtARF1 and AtARF2 are involved in floral initiation and abscission, and rosette leaf senescence [11]. Later research has found that AtARF2 represses the cell division and expansion in many vegetative and floral organs [12]. It may also serve as a molecular link with ABA-mediated regulation of seed germination, and root meristem and drought stress [13-15]. AtARF3 has confirmed the roles in self-incompatibility, gynoecium patterning, shoot apical meristem maintenance, organ polarity, and regeneration [7,16-19].

In addition to the functions of organ polarity determination and patterning, recent studies indicated that AtARF3 may have different roles in early flower development [20]. Further gene function analysis revealed that ARF3 controls cell division in the floral meristem by repressing cytokinin biosynthesis and signaling [21]. AtARF5 is reported to regulate gametophyte and embryo development, root growth and leaf vascular differentiation [22-24]. Furthermore, sweet potato ARF5 may participate in carotenoid biosynthesis, salt, and drought tolerance in transgenic Arabidopsis [25]. AtARF7 regulates hypocotyl response to blue light and auxin stimulation, while AtARF8 affects hypocotyl elongation, auxin homeostasis and fruit development [26-28]. In the monocot plant, the first full-length ARF gene was cloned from rice and named ARF1 [29]. The antisense OsARF1 transgenic rice shows extremely low growth in vegetative and was sterile [30]. At present, function studies have revealed the effect of $A R F$ genes in the process of plant growth and development, but there are still many unknown roles. Thus far, compared with other studies, the biological functions of $A R F$ genes in temperature stress response are limited [31,32]. ARF 10/17 genes are induced in anthers of heat stress-insensitive cotton and suppressed in heat stress-sensitive cotton, suggesting the activation of auxin response to anther indehiscence [31]. Microarray and quantitative PCR analyses find that most OsARF genes are suppressed by cold and heat stresses in the leaves of rice seedling [32].

Sorghum belongs to the grass family and is a typical annual $\mathrm{C}_{4}$ plant. To be a worldwide foodstuff and cash crop, it is distributed throughout the tropical, subtropical, and temperate regions [33]. Sorghum also has the characteristics of rapid growth, heat tolerance, and drought tolerance. The previous studies have predicted 25 ARF genes in Sorghum bicolor ( $S b A R F$ ) and indicated that SbARFs may play complex roles in salt and drought stresses [34]. In this study, more effective software (such as Ensembl Plants, MEGA X, MEME, SMART, and InterPro) allow us to reclassify and structure analysis of the ARFs genes in three plants, including sorghum, rice, and Arabidopsis. The detailed information about $S b A R F$ genes was provided on sequence structures, characteristic regions, and motif compositions. And we focused on the expression pattern of these SbARF genes during development and temperature stress in sorghum. Additionally, in situ hybridization results indicated the location and distribution of SbARF genes under temperature treatments. This study provides a different perspective for future regulation mechanism understanding of the auxin response factors in sorghum development and temperature stress.

\section{Results}

\subsection{Identification of SbARF Genes}

The previous studies have predicted 25 ARF genes in Sorghum bicolor and divided into four distinct classes. To verify the SbARF genes, BLASTP approaches were employed for the mining of all putative $S b A R F$ members in the Sorghum bicolor genome. Finally, a total of 25 potential SbARF genes were identified which have the same sequence ID as described before (Table 1). Further analysis of the 25 SbARF genes revealed that the ORF length of this family ranged between 1557 (SbARF16) and 3432 (SbARF7/25) bp, with a mean length of $2389 \mathrm{bp}$, while the transcript length ranged between 1869 (SbARF2) and 3836 (SbARF7) bp, with a mean length of $2763 \mathrm{bp}$. The predicted polypeptide length ranged between 518 (SbARF16) and 1143 (SbARF7/25) amino acids, with a mean length of 
795 amino acids. The isoelectric point of the ARF proteins ranged between 5.7 (SbARF8) and 8.7 (SbARF2), with a mean value of 6.8. The predicted molecular mass of the proteins ranged between 56 (SbARF16) and 127 (SbARF7) kDa, with a mean molecular mass of $88 \mathrm{kDa}$.

\subsection{Phylogenetic Analysis and Classification of SbARF Genes}

A phylogenetic tree without roots was generated based on multiple alignments of SbARFs with their orthologs from rice and Arabidopsis (Figure 1). All 73 ARFs (including 25 SbARFs, 25 OsARFs, 23 AtARFs) fell into five broad groups: Class I, II, III, IV, and V, which contain 23, 25, 10, 11, and four members, respectively. Obviously, most ARFs of the three species were clustered together in Class I and II while Class V contained the fewer members of ARFs. Thus, the $25 \mathrm{SbARFs}$ could also be divided into five separate clusters as well. Class I included SbARF 7/8/14/15/17/19/22/23/25; Class II included SbARF 3/6/9/11/12/18; Class III included SbARF 4/5/20/21; Class IV included SbARF 1/2/10/13/24; Class V only contained one member, SbARF 16. Furthermore, nearly all SbARF were more closely related to rice than Arabidopsis.

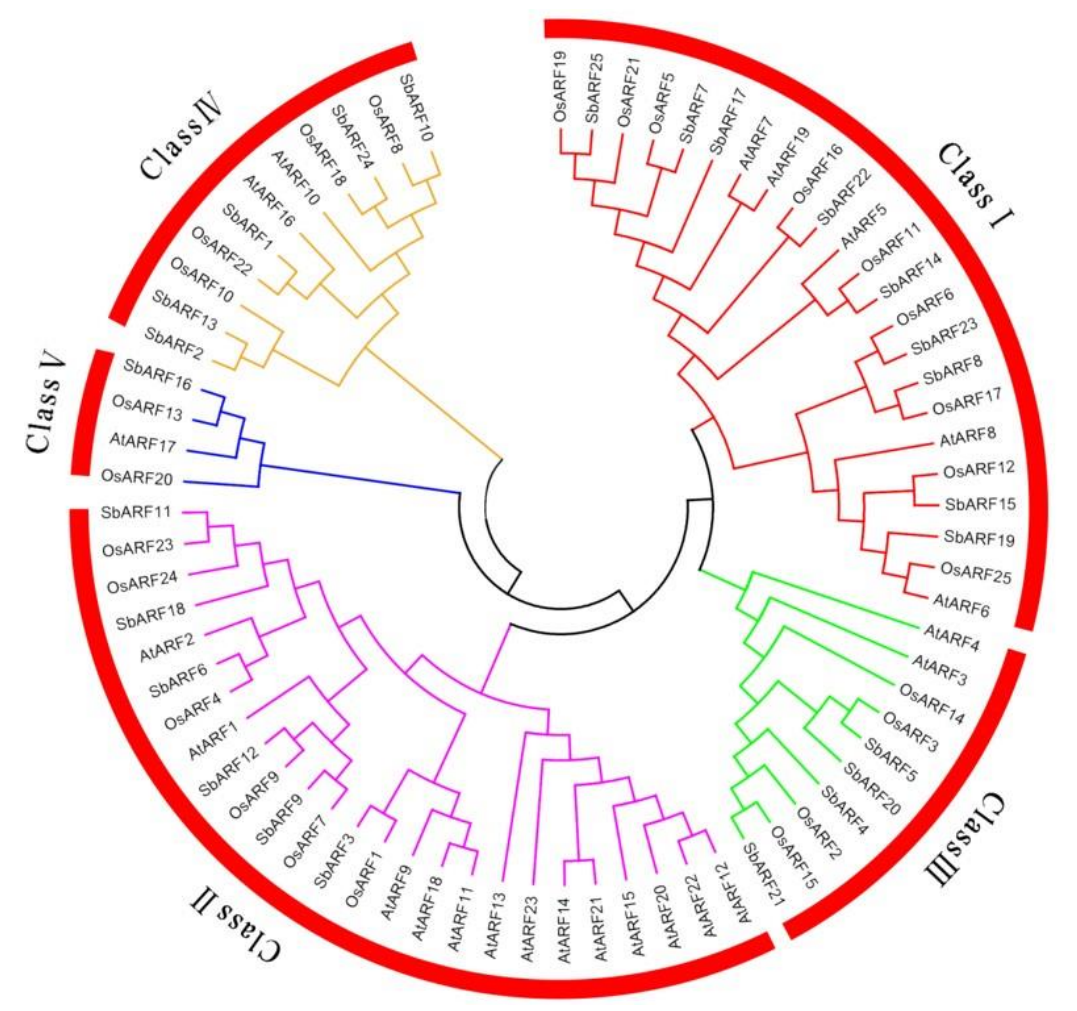

Figure 1. Phylogenetic analysis of ARF in Sorghum bicolor, Oryza sativa and Arabidopsis. The NJ tree shows that the ARFs of Sorghum bicolor (25), Oryza sativa (25), and Arabidopsis (23) were clustered into five classes (I-V). Class I, II, III, IV, and V are marked with red, fuchsia, green, yellow, and blue lines, respectively. 
Table 1. SbARF gene family information.

\begin{tabular}{|c|c|c|c|c|c|c|c|c|c|c|c|c|}
\hline $\begin{array}{c}\text { SbARF } \\
\text { gene }\end{array}$ & Sequence ID & Chr.Location & $\begin{array}{c}\text { Strand } \\
\text { Direction }\end{array}$ & $\begin{array}{c}\text { ORF } \\
\text { Length (bp) }\end{array}$ & $\begin{array}{c}\text { Transcript } \\
\text { Length (bp) }\end{array}$ & $\begin{array}{c}\text { No. } \\
\text { Exons }\end{array}$ & $\begin{array}{l}\text { No. Coding } \\
\text { Exons }\end{array}$ & UniProt & $\begin{array}{c}\text { Protein } \\
\text { Length (aa) }\end{array}$ & MW (Da) & PI & Domains \\
\hline SbARF1 & Sb01g019130.1 & Chr1:20265083-20269209 & Reverse & 2070 & 2354 & 3 & 3 & C5WYD5 & 689 & $75,404.92$ & 6.9 & DBD, MR, PB1 \\
\hline SbARF2 & Sb02g032210.1 & Chr2:66973093-66975465 & Reverse & 1869 & 1869 & 4 & 4 & C5X7P6 & 622 & $68,290.21$ & 8.7 & $\mathrm{DBD}, \mathrm{MR}, \mathrm{PB} 1$ \\
\hline SbARF3 & Sb03g000530.1 & Chr3:375293-379543 & Reverse & 2067 & 2712 & 14 & 14 & C5XJJ7 & 688 & $76,895.05$ & 6.4 & DBD, MR, PB1 \\
\hline SbARF4 & Sb03g030740.1 & Chr3:58980057-58985203 & Reverse & 2109 & 2418 & 11 & 10 & C5XG18 & 702 & $77,003.66$ & 6.4 & $\mathrm{DBD}, \mathrm{MR}$ \\
\hline SbARF5 & Sb03g034850.1 & Chr3:62997617-63003214 & Reverse & 2058 & 2584 & 10 & 10 & - & 685 & $74,377.98$ & 7.2 & DBD, MR \\
\hline SbARF6 & Sb03g044630.1 & Chr3:71975563-71980937 & Forward & 2115 & 2729 & 13 & 13 & C5XH00 & 704 & $78,702.14$ & 7.4 & $\mathrm{DBD}, \mathrm{MR}$ \\
\hline SbARF7 & Sb04g003240.1 & Chr4:3072716-3080254 & Reverse & 3432 & 3836 & 15 & 14 & C5XUJ9 & 1143 & $127,041.26$ & 6.3 & $\begin{array}{l}\text { DBD, MR, } \\
\text { CTD, PB1 }\end{array}$ \\
\hline SbARF8 & Sb04g004430.1 & Chr4:4219215-4225954 & Forward & 2736 & 3126 & 15 & 14 & C5XVH8 & 911 & $100,417.56$ & 5.7 & $\begin{array}{l}\text { DBD, MR, } \\
\text { CTD, PB1 }\end{array}$ \\
\hline SbARF9 & Sb04g022830.1 & Chr4:52419119-52425028 & Reverse & 2019 & 2809 & 15 & 14 & C5XUU5 & 672 & $74,930.18$ & 6.2 & $\begin{array}{l}\text { DBD, MR, } \\
\text { CTD, PB1 }\end{array}$ \\
\hline SbARF10 & Sb04g026610.1 & Chr4:56508838..56511492 & Forward & 2127 & 2487 & 3 & 3 & C5XXU7 & 708 & $76,087.88$ & 7.1 & DBD, MR, PB1 \\
\hline SbARF11 & Sb05g019540.1 & Chr5:47874380-47882162 & Reverse & 2568 & 3071 & 15 & 14 & - & 855 & $93,782.34$ & 6.5 & $\begin{array}{l}\text { DBD, MR, } \\
\text { CTD, PB1 }\end{array}$ \\
\hline SbARF12 & Sb06g017490.1 & Chr6:46853929-46861508 & Forward & 1986 & 3151 & 14 & 14 & C5Y8U9 & 661 & $73,201.76$ & 6.1 & $\begin{array}{l}\text { DBD, MR, } \\
\text { CTD, PB1 }\end{array}$ \\
\hline SbARF13 & Sb06g022810.1 & Chr6:52026442-52029170 & Forward & 2088 & 2088 & 4 & 4 & C5YCE3 & 695 & $75,065.79$ & 8.2 & DBD, MR, PB1 \\
\hline SbARF14 & Sb06g031900.1 & Chr6:60209330-60214539 & Reverse & 2841 & 2841 & 13 & 13 & C5Y9Z0 & 946 & $103,749.51$ & 6.2 & $\begin{array}{l}\text { DBD, MR, } \\
\text { CTD, PB1 }\end{array}$ \\
\hline SbARF15 & Sb06g032500.1 & Chr6:60654734-60662333 & Forward & 2466 & 3084 & 16 & 14 & C5YA53 & 821 & $90,757.87$ & 6.5 & $\begin{array}{l}\text { DBD, MR, } \\
\text { CTD, PB1 }\end{array}$ \\
\hline SbARF16 & Sb06g033970.1 & Chr6:61857871-61861319 & Reverse & 1557 & 1928 & 3 & 2 & C5YB36 & 518 & $56,284.76$ & 6 & DBD, MR \\
\hline SbARF17 & Sb07g027080.1 & Chr7:62218509-62225164 & Reverse & 3288 & 3716 & 13 & 13 & C5YIB6 & 1095 & $121,248.57$ & 6.5 & $\begin{array}{l}\text { DBD, MR, } \\
\text { CTD, PB1 }\end{array}$ \\
\hline SbARF18 & Sb08g014320.1 & Chr8:37871039-37877027 & Reverse & 2520 & 3050 & 15 & 14 & C5YNM8 & 839 & $92,454.03$ & 6.7 & $\begin{array}{l}\text { DBD, MR, } \\
\text { CTD, PB1 }\end{array}$ \\
\hline SbARF19 & Sb08g021460.1 & Chr8:53122868-53128155 & Reverse & 2688 & 3063 & 14 & 14 & C5YRZ9 & 895 & $98,609.5$ & 5.9 & $\begin{array}{l}\text { DBD, MR, } \\
\text { CTD, PB1 }\end{array}$ \\
\hline SbARF20 & Sb09g025500.1 & Chr9:54931364-54935901 & Forward & 1728 & 2002 & 9 & 8 & C5Z0X8 & 575 & $63,169.94$ & 7.5 & $\mathrm{DBD}, \mathrm{MR}$ \\
\hline SbARF21 & Sb09g028450.1 & Chr9:57360258-57365448 & Forward & 2220 & 2483 & 12 & 11 & C5YVJ4 & 739 & $80,854.09$ & 7.7 & DBD, MR \\
\hline SbARF22 & Sb10g006440.1 & Chr10:5930430-5936935 & Forward & 2859 & 2859 & 14 & 14 & C5Z600 & 952 & $105,068.55$ & 6.4 & $\begin{array}{l}\text { DBD, MR, } \\
\text { CTD, PB1 }\end{array}$ \\
\hline SbARF23 & Sb10g027220.1 & Chr10:56924176-56929286 & Reverse & 2760 & 2760 & 14 & 14 & C5Z7U5 & 919 & $101,692.05$ & 6.4 & $\begin{array}{l}\text { DBD, MR, } \\
\text { CTD, PB1 }\end{array}$ \\
\hline SbARF24 & Sb10g027790.1 & Chr10:57623853-57627338 & Forward & 2130 & 2493 & 4 & 3 & C5Z8A5 & 709 & $76,700.16$ & 7.7 & $\mathrm{DBD}, \mathrm{MR}, \mathrm{PB} 1$ \\
\hline SbARF25 & Sb10g029130.1 & Chr10:58946685-58953873 & Forward & 3432 & 3574 & 14 & 14 & C5Z981 & 1143 & $126,297.21$ & 6.6 & $\begin{array}{l}\text { DBD, MR, } \\
\text { CTD, PB1 }\end{array}$ \\
\hline
\end{tabular}




\subsection{Gene Structure and Protein Structure of SbARF Genes}

To better understanding, the structure of SbARF genes, their intron/exon number, positions, and functional domains were analyzed. As shown in Table 1 and Figure 2, the number of exons in DNA sequences varied from 3 to 16 , while the number of coding exons varied from 2 to 14 . All SbARFs had two characteristic regions: a highly conserved region corresponding to the B3-type DNA-bind domain (DBD) in the N-terminal portion, and a middle region that functions as activation or repression domain (MR). Thirteen of which contained the C-terminal Aux/IAA domains (CTD). Nineteen of which contained the type I/II Phox and Bem1p protein-protein interaction domains (PB1). In general, the $S b A R F$ genes from the same clusters shared a similar structure. For example, polypeptide length of Class I was the longest, with a mean length of 981 amino acids. Members of Class I and Class II had 13 or 14 coding exons. ARF genes from other classes contained fewer exons and encoded proteins of shorter lengths. All proteins from Class I had four characteristic regions (DBD, MR, CTD, PB1), while proteins from Class III, IV, V had only two characteristic regions (DBD, MR).
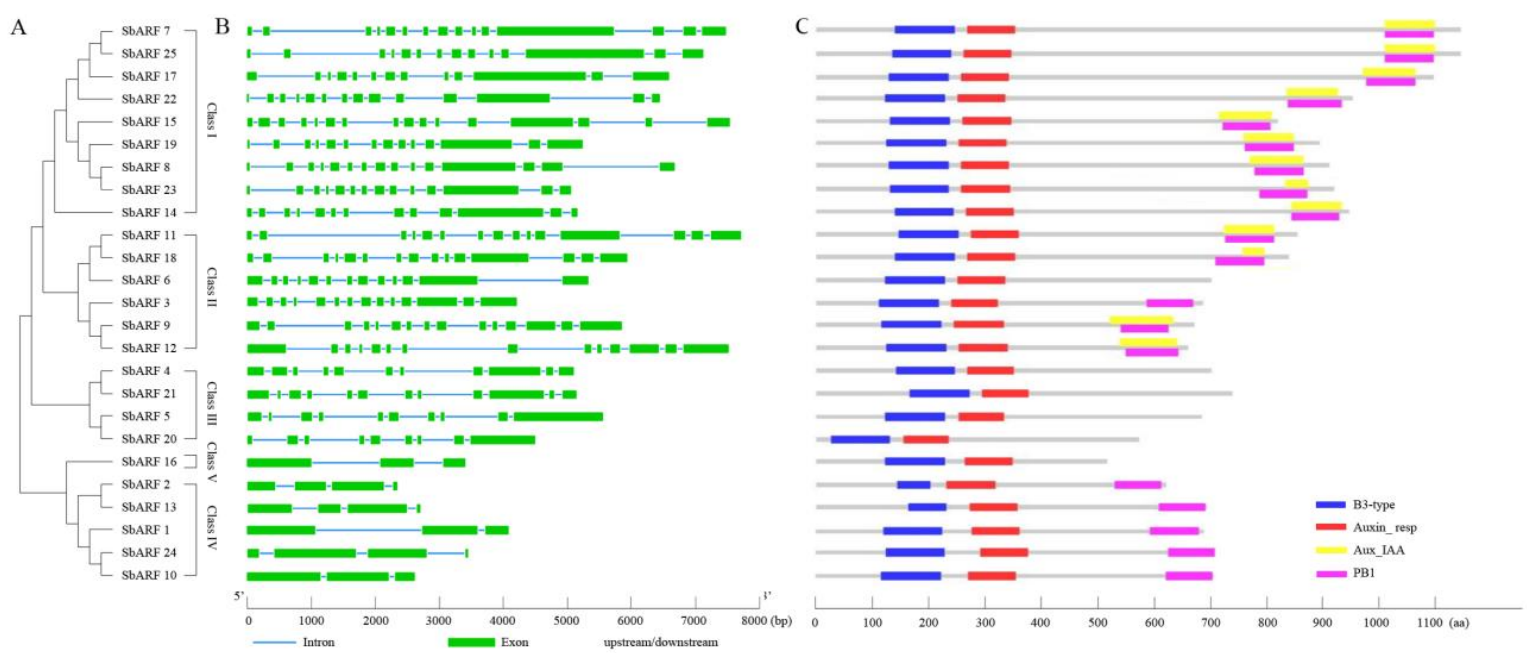

Figure 2. ARF gene family in Sorghum bicolor. (A). Multiple alignments of 25 SbARFs using Clustal $\mathrm{W} 1.83$. The phylogenetic tree was constructed using MEGA X software with a bootstrap analysis of 1000 replicates. (B) Exon/intron distribution in the SbARF family genes. (C) Conserved domain analysis of SbARFs. The length of exons/intron and conserved domain can be estimated using the scale at the bottom, respectively.

A conserved motif analysis of $25 \mathrm{SbARF}$ protein sequences using the MEME online tool predicted 20 motifs (Figure 3). The results displayed, the closer they were in the evolution tree, the more similar location of the motifs would be. Motif 1 was the most conserved motifs with an e-value of 9.6E-1367, and 23 SbARFs had this motif. Twenty-five SbARFs had motif 2, while this motif was also very conservative with the e-value of $7.2 \times 10^{-544}$ (Table S3). Besides these, $24 \mathrm{SbARFs}$ contained motifs 3/4/5/8/9/10. Three characteristic domains (DBD, MR, CTD) of SbARFs were divided into 10 motifs. Motif 1 and 9 constituted the DBD. The MR domain consisted of motifs 2, 4, 8, and 10. The CTD corresponded to motifs 6, 7, and 11. Additionally, all the SbARFs were predicted to be nuclear localized using UniProt online analysis tool and subCELlular LOcalization predictor. 


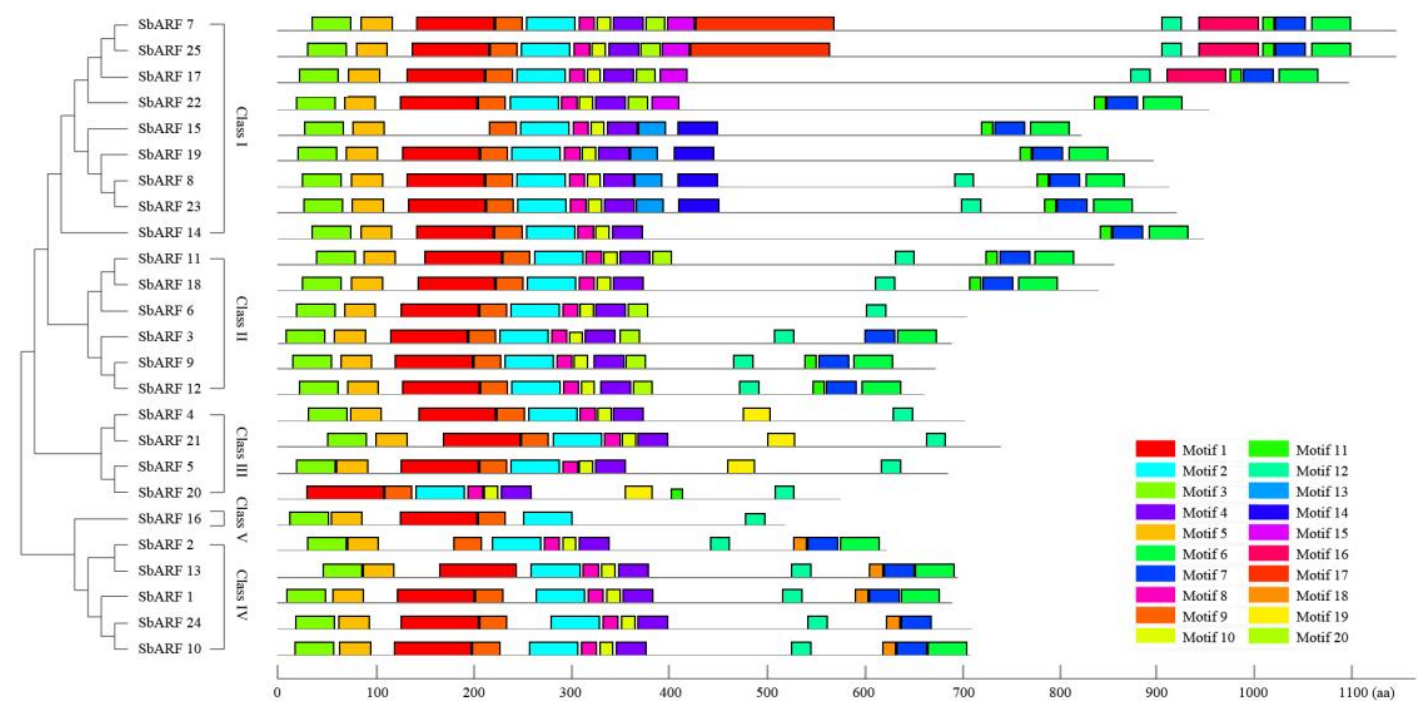

Figure 3. Motif analysis of the SbARF family proteins. Each colored box represents a motif of the protein. The sequence information of 20 motifs is provided in Table S3. The length of proteins can be estimated using the scale at the bottom. The clusters' information is marked on the left.

\subsection{Expression of SbARF Genes in Different Organs and Tissues}

Specific primers were designed to amplify 25 ARF genes in Sorghum bicolor. The expression levels of SbARF genes were analyzed using qRT-PCR in different tissues including vegetative organs (mature leaves, roots, stems) and reproductive organs (immature inflorescence, mature inflorescence, post-flowering inflorescence, and mature seeds) (Figure 4). Generally, most SbARF genes showed low expression in mature stems, and high expression in different tissues, except that $S b A R F 2$ and SbARF16 exhibited low transcript levels in all the collected tissues. Some SbARF genes including $S b A R F 3 / 5 / 6 / 7 / 8 / 9 / 12 / 13 / 15 / 17 / 19 / 20$ showed special higher expressions in the vegetative organs, while $S b A R F 4 / 10 / 14$ showed special higher expressions in the reproductive organs. Among these highly expressed genes (15), most of them from Class I (6), Class II (4) and Class III (3). Additionally, transcript accumulation could be detected for several SbARF genes (SbARF1/11/16/21/22/24/25) in both vegetative and reproductive organs. For example, SbARF3/12/13/15/16/F20 genes were expressed more strongly in the leaves than in the other organs. Ten SbARF genes (SbARF5/6/7/8/9/17/18/19/23/F24) were strongly induced in the roots. SbARF14 and SbARF 22 genes were remarkably enhanced in mature seeds, while SbARF4/25 and SbARF10 genes displayed higher expression in mature inflorescence and post-flowering inflorescence, respectively. 


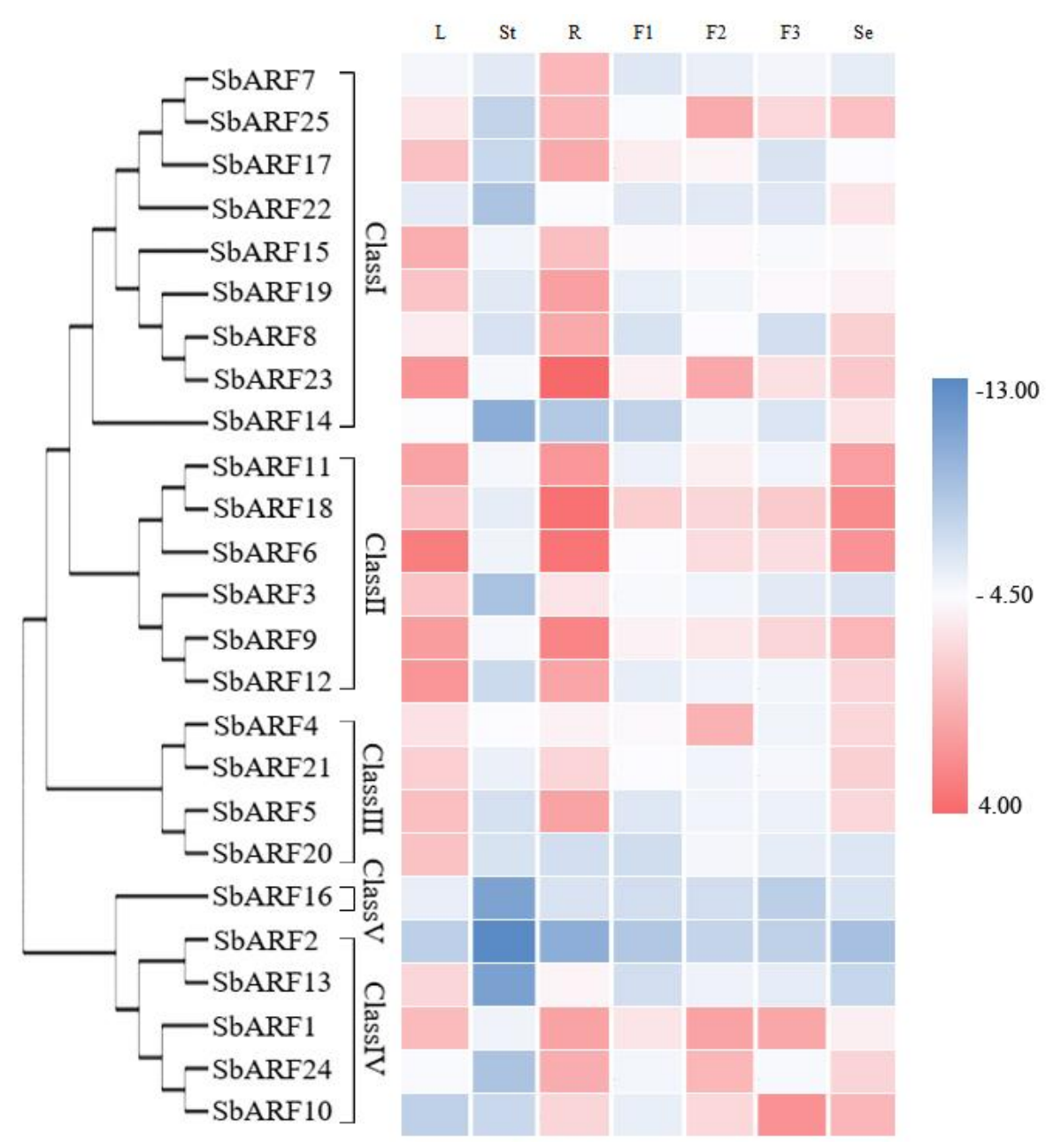

Figure 4. Expression profiles of $S b A R F$ genes in different tissues. The heatmap was generated based on the relative expression values of 25 SbARFs genes obtained by qRT-PCR in seven different tissues and organs. The red and blue color scale represents log2-transformed values and indicates relatively high or low expression, respectively. Every sample is three biological replicates. L, leaves; St, stem; R, root; F1, immature inflorescence; F2, mature inflorescence; F3, post-flowering inflorescence; Se, seeds.

\subsection{Expression of SbARF Genes in Response to Temperature Stress}

As transcription factors, ARFs could modulate auxin signaling pathway, and then controlled a wide array of plant behaviors including developmental processes and abiotic stress response. To further determine the role of $S b A R F$ genes in temperature stress response, expression characteristics of $S b A R F$ genes were investigated in seedling under $4{ }^{\circ} \mathrm{C}$ and $40{ }^{\circ} \mathrm{C}$ treatment. As shown in Figure 5 and Figure S1, the expressions of $9 S b A R F$ genes ( $S b A R F 4 / 7 / 9 / 15 / 17 / 19 / 21 / 22 / 24)$ were always up-regulated after cold and heat stress, while SbARF13 remained down-regulated during the whole treatments. Three $S b A R F$ genes ( $S b A R F 1 / 3 / 16)$ showed low expression levels during $4{ }^{\circ} \mathrm{C}$ treatment, but $9 \mathrm{SbARF}$ genes (SbARF5/6/8/10/11/12/18/20/25) displayed different expression patterns. For example, SbARF11 and $S b A R F 25$ were significantly down-regulated after $1 \mathrm{~h}$ of $4{ }^{\circ} \mathrm{C}$ treatment and then were rapidly up-regulated after a $3 \mathrm{~h}$ treatment. Eleven $S b A R F$ genes ( $S b A R F 1 / 5 / 6 / 8 / 10 / 11 / 12 / 16 / 18 / 20 / 25)$ showed high expression levels during $40^{\circ} \mathrm{C}$ treatment, but $S b A R F 3$ was obviously suppressed after $1 \mathrm{~h}$ treatment and then slightly accumulated after $6 \mathrm{~h}$ treatment. It was worth mentioning that the expression of the SbARF23 gene was obvious changed not only at $4{ }^{\circ} \mathrm{C}$ but also at $40{ }^{\circ} \mathrm{C}$ treatments.

Most of the $S b A R F$ genes from Class I and Class III displayed transcript accumulation after temperature stress. Instead, the expression levels of SbARF1/10/13 (Class IV) and SbARF16 (Class V) 
were significantly suppressed after treatment. Furthermore, SbARF13 was the only gene that always maintained a low level.

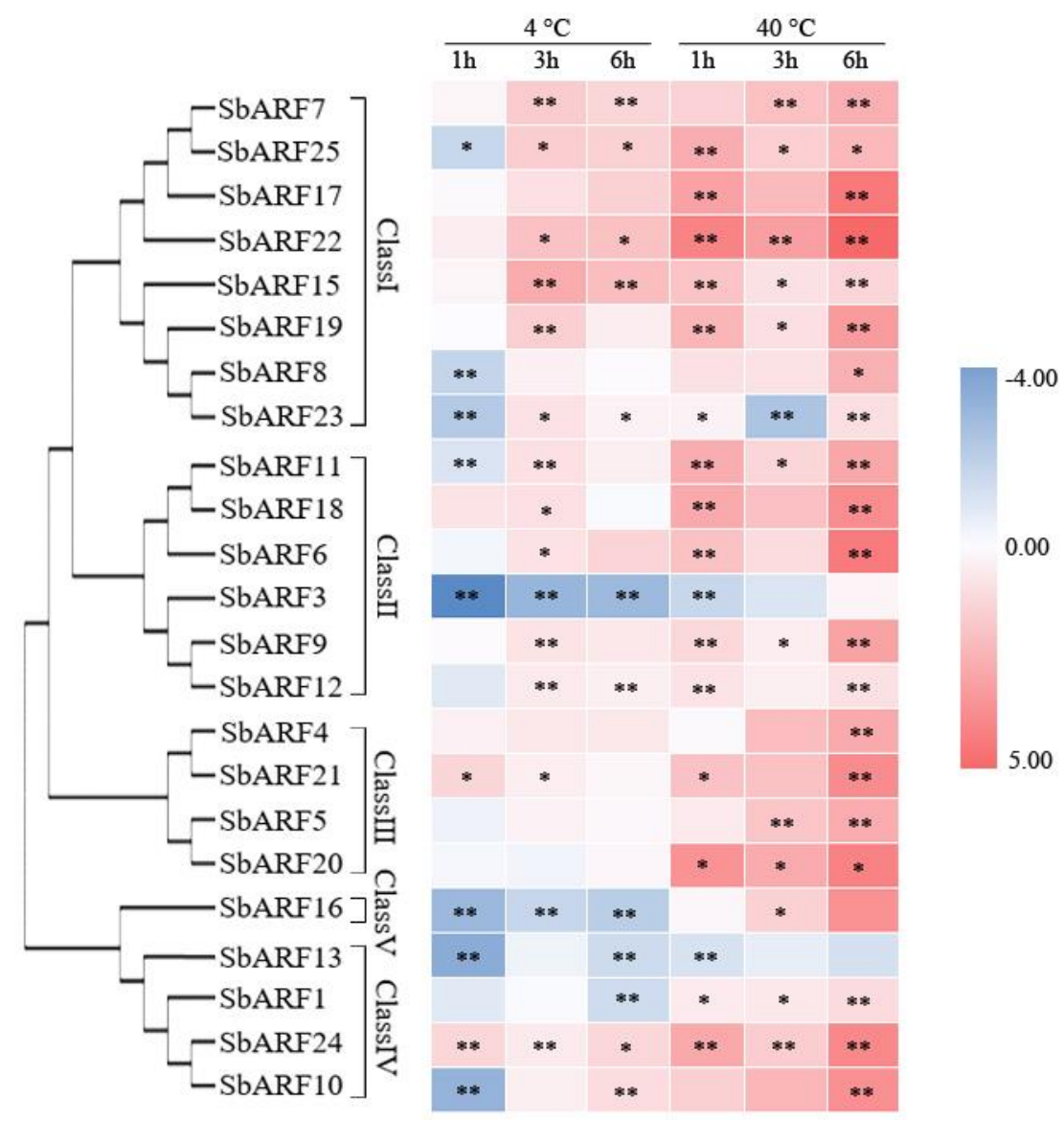

Figure 5. Expression profiles of $S b A R F$ genes in sorghum seedling under cold and heat stress. The heatmap was generated based on the relative expression values of 25 SbARFs genes obtained by qRT-PCR. The expression levels of ARF genes under non-stressed conditions were defined as 1 . The red and blue color scale represents $\log 2$-transformed values and indicates relatively high or low expression, respectively. Data from three biological replicates are displayed with standard deviation. Asterisks on the top of bars indicate significant differences as determined by Student's $t$-test $\left.{ }^{*}: p<0.05 ;{ }^{* *}: p<0.01\right)$.

\subsection{Distribution Patterns of the SbARF Genes under Temperature Stress}

To investigate the tissue site of auxin signal response under temperature stress in Sorghum bicolor, in situ hybridization analysis was used to detect the distribution patterns of SbARF genes in seedling leaves under temperature stress. According to the significant expression change of SbARFs, SbARF3/15/17/24 genes were selected for in situ hybridization analysis. As shown in Figure 6, these $S b A R F$ mRNAs displayed similar accumulation sites in the leaves. The hybridization signals were accumulated in the vascular bundles of the main and secondary veins, especially in the vascular bundle sheath cells and the surrounding parenchyma cells. The other cells were almost non-detected signals. Although the stain signals were weak after cold stress, they followed a similar distribution pattern no matter heat stress or cold stress. 


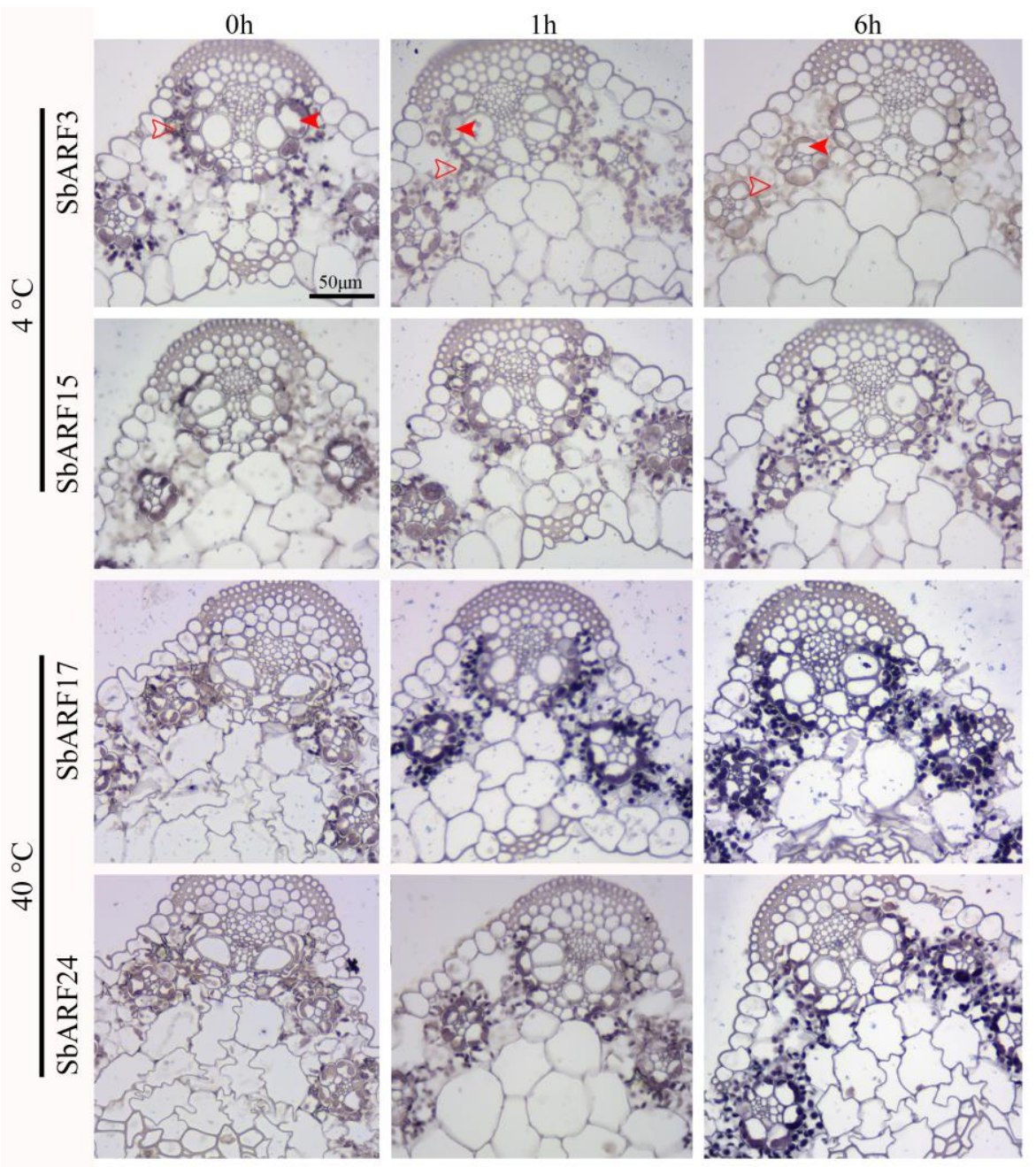

Figure 6. In situ hybridization analysis of $S b A R F$ genes in seedling leaves under temperature stress. Solid and feint red arrows indicate the regions of vascular bundle sheath cells and the surrounding parenchyma cells, respectively.

\section{Discussion}

As a worldwide food and feed cereal crop, sorghum has the characteristics of rapid growth, high-temperature tolerance, and drought tolerance. Here, a total of 25 potential $S b A R F$ genes were identified, which have the same sequence ID as described before (Table 1). There was the same number of SbARF genes as ARF genes encoded in Oryza sativa (25) [6], but more than Arabidopsis (23) [5]. The previous studies have also predicted 25 ARF genes in Sorghum bicolor [34]. It is worth mentioning that effective bioinformatics methods were used to further analyze the sequence feature in our study, such as Ensembl Plants, MEGA X, MEME, SMART, InterPro, and so on.

We found that 73 ARFs (25 SbARFs, 25 OsARFs, and 23 AtARFs) could be categorized into five classes in phylogenetic analyses (Figure 1). Sorghum is an ideal $\mathrm{C}_{4}$ grass model and can be a good complement to $\mathrm{C}_{3}$ rice for biofuel crops, animal feed, and forage. In the evolutionary tree of ARFs, there were also showed a more close relationship between monocotyledon Sorghum bicolor and Oryza sativa. Furthermore, the protein typical domains and conserved motifs analysis showed that SbARFs from the same class might have similar regions (Figures 2 and 3). The typical N-terminal B3-type DNA binding domain (DBD) of ARF protein may recognize and bind to AuxREs in the promoters of auxin response genes [35]. In Sorghum bicolor, all $25 \mathrm{SbARFs}$ had this typical DBD domain, and very conservative. As showing in Figure 3, 23 of those DBD domains were constituted by motif 1 and 9. Only ARF2 and ARF13 (members of Class IV) were different because their DBD domains were constituted by motif 9 or 
motif 2, respectively. Besides the DBD domain, 25 SbARFs also had an Auxin_resp middle region (MR), and most of the MR regions were constituted by motif 2, 4, 8, and 10. Only SbARF16 was different because its MR region was constituted by motif 2 . The MR region may act as an activation domain (AD) or repression domain (RD). Although there may be still some confusion on the mechanisms of activation and repression, the ADs are found to be enriched in glutamine $(\mathrm{Q})$, while the RDs are enriched in serine (S), proline $(\mathrm{P})$, and threonine $(\mathrm{T})[10,35,36]$. Interestingly, motifs $2,4,8$, and 10 in SbARFs contained amino acids $\mathrm{S}, \mathrm{T}$, and $\mathrm{P}$, but not $\mathrm{Q}$. The amino acid composition of MR may contribute to further SbARF functional annotation.

C-terminal domain (CTD) was the third characteristic domain in ARF proteins. It has consisted of a dimerization domain (domain III/IV) and involved in protein-protein interactions by dimerizing with proteins encoded by genes from Aux/IAA family or even dimerizing between ARFs [1,3,4]. However, only 13 of 25 SbARFs (members of Class I and II) contain the typical AUX_IAA domains, while 19 of $25 \mathrm{SbARFs}$ contain a type I/II Phox and Bem1p protein-protein interaction domain (PB1) (Table 1 and Figure 2). Furthermore, The PB1 domains overlapped with the AUX_IAA domains in those 13 SbARFs. Recently researches suggest that PB1 domain can interact with other PB1 via electrostatic interactions, and provides positive and negative interfaces. The underlying protein interaction between ARFs and Aux/IAA also contains a PB1 domain [35,36].

The transcript expression profiles can help us screen for candidate $S b A R F$ genes with potentially distinct functions. The transcriptional abundance of the SbARF genes varied greatly in different vegetative organs and reproductive organs (Figure 2), suggesting that the SbARF genes had multiple functions in sorghum growth and development. According to the evolutionary tree analysis, Class II members (SbARF11/18, SbARF9/12) with the highest homology showed the most similar expression patterns, followed by Class I (SbARF7/25, SbARF8/23), and other classes had lower similarity, indicating potential functional redundancy in Class I and Class II members. Eleven of 25 SbARF genes were accumulated in the roots than other tissues, with five (SbARF7/8/17/19/23) in Class I and four (SbARF6/9/11/18) in Class II, SbARF5 in Class III and SbARF24 in Class IV. Seven of 25 SbARF genes were accumulated in the leaves, with SbARF15 in class I, SbARF3/12 in Class II, SbARF20/21 in Class III, SbARF13 in Class IV, SbARF16 in Class V. SbARF4 (Class III) and SbARF25(Class I) genes were highly induced in the mature inflorescence. SbARF10 gene (Class IV) was remarkably increased in the post-flowering inflorescence. SbARF14 and SbARF22 genes (both members of Class I) were highly expressed in the seeds than other tissues. SbARFs shared remarkable homology with OsARFs as showed in Figure 1. It has been reported that the transgenic antisense-OsARF1 rice grew slowly and leaves were short and curly [30], while its sorghum homolog, SbARF3, was prominently higher expressed in the leaves, implying its possible function in vegetative development. The knockout mutant of OsARF12 and double mutant of OsARF12/25 display unusual growth of seedling, especially in root growth $[37,38]$. Thus, their sorghum homologs SbARF15 and SbARF19 genes may be important for vegetative growth in sorghum, along with obviously higher accumulation in both leaves and roots, but lower in reproductive organs. However, different from its rice homolog OsARF16 gene, which is required for auxin responses in the roots [39], the expression of the SbARF22 gene peaked in the mature seeds, and then in the roots. The evidence of the similarities and differences between the transcription levels may imply the evolution and divergence of Sorghum bicolor and Oryza sativa in some way.

Sorghum is a typical $C_{4}$ grass model crop adapted to heat stress [33]. Auxin is well-known for its numerous functions in plant morphogenesis and plant tolerance to stresses, including heat stress and cold stress [31,32]. It was reported that ARF10 and ARF17 genes are induced in anthers of heat stress-insensitive cotton and suppressed in heat stress-sensitive cotton [31]. In rice, microarray and quantitative PCR analyses find that most OSARF genes are suppressed by cold and heat stresses, while OsARF4/14/18/19 and OsARF11/13/16 are induced by cold and heat stresses, respectively [32]. It's worth mentioning that their sorghum homologs $S b A R F 6 / 24 / 25$ and $S b A R F 16 / 22$ also were significantly induced by cold and heat stresses, respectively. Furthermore, $S b A R F$ genes with the highest homology in Class I, Class II, and Class III showed similar accumulation pattern in normal growth and development, 
except members in Class IV and Class V. In temperature stress, members of Class I and Class III showed similar accumulation pattern, while some members of Class II and Class IV had different expression patterns. The finding also supported the potential functional redundancy in Class I members and no only that, those genes (such as members in Class I/II/IV/V) that the expression levels changed significantly may have special roles in temperature stress. Clearly, most of $S b A R F$ genes were induced by heat stress, which possibly indicated the participation of auxin in the high temperature resistant of sorghum.

Since ARFs are important proteins in auxin-mediated response, which effect the activation of Aux/IAA and PIN, the in situ hybridization might point out the auxin response site in tissues under temperature stress. We found that no matter heat or cold stress, the distribution patterns of $S b A R F$ mRNAs were similar in the seedling leaves (Figure 6). They mainly expressed in the vascular bundle sheath cells and the surrounding parenchyma cells. The distribution pattern of vascular tissue accumulation is consistent with the accumulation of PINs and auxin in the vascular strands of Arabidopsis [40-43]. It is reported that in the loss-of-function and gain-of-function AtARF5 mutation, not only PIN1 expression is changed, but also the vascular tissue formation is abnormal [22,23]. Auxin efflux carrier PIN3/4/6/7/8 can be also detected in the vascular tissues of Arabidopsis, a green signal of PIN3-GFP presents primarily in the xylem parenchyma cells, PIN4/7 expressed in the cambium, PIN6 expressed in the cambial cells adjacent to the xylem, and PIN8 expressed in the cambial cells near PIN6 [41]. It is hypothesized that these SbARF genes have participated in auxin response to temperature stress in the vascular tissues.

\section{Materials and Methods}

\subsection{Identification and Phylogenetic Analysis of SbARF Genes}

The Sorghum bicolor genome was downloaded from the Resources for Plant Comparative Genomics (PlantGDB; Available online: http://www.plantgdb.org/). The BLASTP method was adopted to search for the $S b A R F$ genes and to determine the maximum number of genes. The sequences of 25 Oryza sativa $A R F$ and 23 Arabidopsis ARF genes were downloaded from the Plant Transcription Factor Database (PlantTFDB; Available online: http://planttfdb.cbi.pku.edu.cn/) [5,6]. These ARFs were used as bait to identify SbARF, via a BLASTP search at the score value of $\geq 100$ and e-value $\leq 1 \times 10^{-5}$. Then all identified potential ARFs were further validated and analyzed by Ensembl Plants (available online: http://plants.ensembl.org/) and PFAM database (available online: https://www.pfam.xfam.org/). Finally, $25 \mathrm{SbARFs}$ genes were identified for further analysis. The protein sequences were investigated by multiple alignment analyses using Clustal $\mathrm{W}$. The resultant sequence alignment then served as input data for a neighbor-joining phylogenetic tree constructed using the MEGA X software [44] with a bootstrap analysis of 1000 replicates.

\subsection{Gene Structure and Protein Structure Analysis}

The mRNA sequences of SbARF genes were obtained from PlantTFDB. The DNA sequence information and protein information were predicted online using Ensembl Plants and SMART (available online: http://smart.embl-heidelberg.de/). The conserved motifs were predicted by the MEME website (available online: http://alternate.meme-suite.org/tools/meme) [45] with the following parameters: optimum motif width was 6 to 200, and the maximum number of motifs was 20. The InterPro online program (available online: https://www.ebi.ac.uk/interpro/) was used to further analyze and annotate the protein sequences. To predict the function and subcellular location of SbARFs, the UniProt online analysis tool (available online: https://www.uniprot.org/) and CELLO v.2.5: subCELlular LOcalization predictor (available online: http://cello.life.nctu.edu.tw/) were used. 


\subsection{Plant Materials and Treatment Methods}

The tissues and organs from the adult Sorghum bicolor used for gene expression analysis were collected in the experiment field at Xidian University (Xi'an, China). Leaves (L), roots (R), stems (St), immature inflorescence (one week before flowering, F1), mature inflorescence (flowers at full bloom, F2), post-flowering inflorescence (one week after flowering, F3), and mature seeds (Se) were collected from seven-week-old plants. The samples were performed according to the previous description [46]. For the temperature stress treatments, sorghum seeds were grown in Murashige and Skoog medium in an illumination incubator at $24 \pm 2{ }^{\circ} \mathrm{C}$ with 16 -h light/8-h dark cycles. Fourteen-day-old seedlings were treated with $40^{\circ} \mathrm{C}$ as heat stress or $4{ }^{\circ} \mathrm{C}$ as cold stress for $1 \mathrm{~h}, 3 \mathrm{~h}$, and $6 \mathrm{~h}$, and then were collected immediately. Each experiment was repeated three times and all the materials were stored at $-80^{\circ} \mathrm{C}$ until RNA extraction.

\subsection{Quantitative RT-PCR Analysis}

Total RNA was extracted using RNAiso Plus (TaKaRa, Tokyo, Japan) according to the manufacturer's instructions, cDNA was synthesized using the Reverse Transcription System (TaKaRa, Tokyo, Japan). Quantitative RT-PCR (qRT-PCR) analysis was performed in a 7300 Real-Time System (ABI, NewYork, American) using the SYBR Green RealMasterMix (TIANGEN, Beijing, China). The qRT-PCR primers were designed by Primer 5 software (Table S1). The housekeeping gene, Actin $\beta$, was used as internal control. The quantification of each sample of cDNA was performed in triplicate, each PCR was replicated three times for verification, and the $2^{-\Delta \Delta C T}$ method was used to analyze the relative changes in gene expression from the qPCR experiments.

\subsection{Paraffin Section of Leaves and in Situ Hybridization}

For fine localization of SbARF mRNA signals in leaves under temperature stress, paraffin section and in situ hybridization analysis were used after $4{ }^{\circ} \mathrm{C}$ and $40{ }^{\circ} \mathrm{C}$ treatments. The 14-day old seedlings of Sorghum bicolor were obtained after $1 \mathrm{~h}$ and $6 \mathrm{~h}$ temperature stress treatments. The leaf tissues were collected and fixed with FAA (70\% alcohol: glacial acetic acid: formalin = 18:1:1, made with diethylpyrocarbonate-treated water) for $1 \mathrm{~h}$. Then the samples were dehydrated and embedded paraffin. The leaf sections were cut at a $10 \mathrm{~mm}$ thickness under a rotary microtome (Leica, Wetzlar, Germany) mounted on poly-Lys-coated glass slides. The gene-specific probe was amplified with the primers Probe-SbARF-F and Probe-SbARF-R (Table S2), and the mouse anti-digoxigenin-labeled alkaline phosphatase (anti-DIG-AP) was added dropwise. Pretreatment, hybridization and immunological detection of sections were performed as described [47].

\section{Conclusions}

The systematic characterization of the $A R F$ gene family in sorghum has revealed key features about their structures and the potential functions of the $S b A R F$ gene family in vegetative and reproductive organs. High and low-temperature treatments and qRT-PCR demonstrated some SbARF genes changed dramatically along with the increase of treatment time. Additionally, in situ hybridization results indicated the location and distribution of $S b A R F$ genes under temperature stress. Our findings provide evidence for future understanding of ARFs in sorghum development and temperature stress.

Supplementary Materials: Supplementary materials can be found at http://www.mdpi.com/1422-0067/20/19/4816/s1.

Author Contributions: D.C. planned and designed the research, analyzed data, and wrote the manuscript. W.W. and Y.W. measured gene expression. L.Z. and H.X. contributed the reagents and analytical tools. Y.Z. and Q.Z. project administration and funding acquisition.

Funding: This study was financially supported by the National Natural Science Foundation of China (nos. 31300268, 81227901, 61471279) and Fundamental Research Funds for the Central Universities (no. JB191209).

Acknowledgments: We would like to acknowledge Haoli Ma and Yujun Ren for revising the manuscript.

Conflicts of Interest: The authors declare no conflict of interest. 


\section{Abbreviations}

$\begin{array}{ll}\text { ARF } & \text { Auxin response factor } \\ \text { qRT-PCR } & \text { Reverse transcription-quantitative PCR } \\ \text { AuxREs } & \text { Auxin-response elements } \\ \text { DBD } & \text { N-terminal B3-type DNA binding domain } \\ \text { AD } & \text { Activation domain } \\ \text { RD } & \text { Repression domain } \\ \text { CTD } & \text { C-terminal domains } \\ \text { Aux/IAA } & \text { Auxin/indole-3-acetic acid } \\ \text { MW } & \text { Molecular weight } \\ \text { PI } & \text { Isoelectric point }\end{array}$

\section{References}

1. Guilfoyle, T.J.; Hagen, G. Auxin response factors. Curr. Opin. Plant Biol. 2007, 10, 453-460. [CrossRef] [PubMed]

2. Liscum, E.; Reed, J.W. Genetics of Aux/IAA and ARF action in plant growth and development. Plant Mol. Biol. 2002, 49, 387-400. [CrossRef] [PubMed]

3. Kim, J.; Harter, K.; Theologis, A. Protein-protein interactions among the Aux/IAA proteins. Proc. Natl. Acad. Sci. USA 1997, 94, 11786-11791. [CrossRef] [PubMed]

4. Piya, S.; Shrestha, S.K.; Binder, B.; Stewart, C.N.; Hewezi, T. Protein-protein interaction and gene co-expression maps of ARFs and Aux/IAAs in Arabidopsis. Front. Plant Sci. 2014, 5, 744. [CrossRef] [PubMed]

5. Ulmasov, T.; Hagen, G.; Guilfoyle, T.J. ARF1, a transcription factor that binds to auxin response elements. Science 1997, 276, 1865-1868. [CrossRef] [PubMed]

6. Wang, D.K.; Pei, K.M.; Fu, Y.P.; Sun, Z.X.; Li, S.J.; Liu, H.Q.; Tang, K.; Han, B.; Tao, Y.Z. Genome-wide analysis of the auxin response factors (ARF) gene family in rice (Oryza sativa). Gene 2007, 394, 13-24. [CrossRef]

7. Liu, Y.; Jiang, H.Y.; Chen, W.J.; Qian, Y.X.; Ma, Q.; Cheng, B.J.; Zhu, S.W. Genome-wide analysis of the auxin response factor (ARF) gene family in maize (Zea mays). Plant Growth Regul 2011, 63, 225-234. [CrossRef]

8. Die, J.V.; Gil, J.; Millan, T. Genome-wide identification of the auxin response factor gene family in Cicer arietinum. BMC Genom. 2018, 19, 301. [CrossRef]

9. Liu, M.Y.; Ma, Z.T.; Wang, A.H.; Zheng, T.R.; Huang, L.; Sun, W.J.; Zhang, Y.J.; Jin, W.Q.; Zhan, J.Y.; Cai, Y.T.; et al. Genome-Wide Investigation of the Auxin Response Factor Gene Family in Tartary Buckwheat (Fagopyrum tataricum). Int. J. Mol. Sci. 2018, 19, 3526. [CrossRef]

10. Zhang, Y.Q.; Zeng, Z.H.; Chen, C.J.; Li, C.Q.; Xia, R.; Li, J.G. Genome-wide characterization of the auxin response factor (ARF) gene family of litchi (Litchi chinensis Sonn.): Phylogenetic analysis, miRNA regulation and expression changes during fruit abscission. Peerj 2019, 7, e6677. [CrossRef]

11. Ellis, C.M.; Nagpal, P.; Young, J.C.; Hagen, G.; Guilfoyle, T.J.; Reed, J.W. AUXIN RESPONSE FACTOR1 and AUXIN RESPONSE FACTOR2 regulate senescence and floral organ abscission in Arabidopsis thaliana. Development 2005, 132, 4563-4574. [CrossRef] [PubMed]

12. Schruff, M.C.; Spielman, M.; Tiwari, S.; Adams, S.; Fenby, N.; Scott, R.J. The AUXIN RESPONSE FACTOR 2 gene of Arabidopsis links auxin signalling, cell division, and the size of seeds and other organs. Development 2006, 133, 251-261. [CrossRef] [PubMed]

13. Wang, L.; Hua, D.P.; He, J.N.; Duan, Y.; Chen, Z.Z.; Hong, X.H.; Gong, Z.Z. Auxin Response Factor2 (ARF2) and its regulated homeodomain gene HB33 mediate abscisic acid response in Arabidopsis. PLoS Genet. 2011, 7, e1002172. [CrossRef] [PubMed]

14. Meng, L.S.; Wang, Z.B.; Yao, S.Q.; Liu, A.Z. The ARF2-ANT-COR15A gene cascade regulates ABA-signaling-mediated resistance of large seeds to drought in Arabidopsis. J. Cell Sci. 2015, 128, 3922-3932. [CrossRef] [PubMed]

15. Promchuea, S.; Zhu, Y.J.; Chen, Z.Z.; Zhang, J.; Gong, Z.Z. ARF2 coordinates with PLETHORAs and PINs to orchestrate ABA-mediated root meristem activity in Arabidopsis. J. Integr. Plant Biol. 2017, 59, 30-43. [CrossRef]

16. Nemhauser, J.L.; Feldman, L.J.; Zambryski, P.C. Auxin and ETTIN in Arabidopsis gynoecium morphogenesis. Development 2000, 127, 3877-3888. [PubMed] 
17. Chitwood, D.H.; Nogueira, F.T.S.; Howell, M.D.; Montgomery, T.A.; Carrington, J.C.; Timmermans, M.C.P. Pattern formation via small RNA mobility. Gene. Dev. 2009, 23, 549-554. [CrossRef]

18. Tantikanjana, T.; Nasrallah, J.B. Non-cell-autonomous regulation of crucifer self-incompatibility by Auxin Response Factor ARF3. Proc. Natl. Acad. Sci. USA 2012, 109, 19468-19473. [CrossRef]

19. Cheng, Z.J.; Wang, L.; Sun, W.; Zhang, Y.; Zhou, C.; Su, Y.H.; Li, W.; Sun, T.T.; Zhao, X.Y.; Li, X.G.; et al. Pattern of Auxin and Cytokinin Responses for Shoot Meristem Induction Results from the Regulation of Cytokinin Biosynthesis by AUXIN RESPONSE FACTOR3. Plant Physiol 2013, 161, 240-251. [CrossRef]

20. Zheng, Y.; Zhang, K.; Guo, L.; Liu, X.; Zhang, Z. AUXIN RESPONSE FACTOR3 plays distinct role during early flower development. Plant Signal. Behav. 2018, 13, e1467690. [CrossRef]

21. Zhang, K.; Wang, R.Z.; Zi, H.L.; Li, Y.P.; Cao, X.W.; Li, D.M.; Guo, L.; Tong, J.H.; Pan, Y.Y.; Jiao, Y.L.; et al. AUXIN RESPONSE FACTOR3 regulates floral meristem determinacy by repressing cytokinin biosynthesis and signaling. Plant Cell 2018, 30, 324-346. [CrossRef] [PubMed]

22. Hardtke, C.S.; Berleth, T. The Arabidopsis gene MONOPTEROS encodes a transcription factor mediating embryo axis formation and vascular development. EMBO J. 1998, 17, 1405-1411. [CrossRef] [PubMed]

23. Krogan, N.T.; Ckurshumova, W.; Marcos, D.; Caragea, A.E.; Berleth, T. Deletion of MP/ARF5 domains III and IV reveals a requirement for Aux/IAA regulation in Arabidopsis leaf vascular patterning. New Phytol. 2012, 194, 391-401. [CrossRef] [PubMed]

24. Liu, Z.N.; Miao, L.M.; Huo, R.X.; Song, X.Y.; Johnson, C.; Kong, L.J.; Sundaresan, V.; Yu, X.L. ARF2-ARF4 and ARF5 are essential for female and male gametophyte development in Arabidopsis. Plant Cell Physiol. 2018, 59, 179-189. [CrossRef] [PubMed]

25. Kang, C.; He, S.Z.; Zhai, H.; Li, R.J.; Zhao, N.; Liu, Q.C. A sweetpotato auxin response factor gene (IbARF5) is involved in carotenoid biosynthesis and salt and drought tolerance in transgenic Arabidopsis. Front. Plant Sci. 2018, 9, 1307. [CrossRef] [PubMed]

26. Harper, R.M.; Stowe-Evans, E.L.; Luesse, D.R.; Muto, H.; Tatematsu, K.; Watahiki, M.K.; Yamamoto, K.; Liscum, E. The NPH4 locus encodes the auxin response factor ARF7, a conditional regulator of differential growth in aerial Arabidopsis tissue. Plant Cell 2000, 12, 757-770. [CrossRef] [PubMed]

27. Goetz, M.; Vivian-Smith, A.; Johnson, S.D.; Koltunow, A.M. AUXIN RESPONSE FACTOR8 is a negative regulator of fruit initiation in Arabidopsis. Plant Cell 2006, 18, 1873-1886. [CrossRef]

28. Tian, C.; Muto, H.; Higuchi, K.; Matamura, T.; Tatematsu, K.; Koshiba, T.; Yamamoto, K.T. Disruption and overexpression of auxin response factor 8 gene of Arabidopsis affect hypocotyl elongation and root growth habit, indicating its possible involvement in auxin homeostasis in light condition. Plant J. 2004, 40, 333-343. [CrossRef]

29. Waller, F.; Furuya, M.; Nick, P. OsARF1, an auxin response factor from rice, is auxin-regulated and classifies as a primary auxin responsive gene. Plant Mol. Biol. 2002, 50, 415-425. [CrossRef]

30. Attia, K.A.; Abdelkhalik, A.F.; Ammar, M.H.; Wei, C.; Yang, J.; Lightfoot, D.A.; El-Sayed, W.M.; El-Shemy, H.A. Antisense Phenotypes Reveal a Functional Expression of OsARF1, an Auxin Response Factor, in Transgenic Rice. Curr. Issues Mol. Biol 2009, 11, I29-I34.

31. Ding, Y.H.; Ma, Y.Z.; Liu, N.A.; Xu, J.; Hu, Q.; Li, Y.Y.; Wu, Y.L.; Xie, S.; Zhu, L.F.; Min, L.; et al. microRNAs involved in auxin signalling modulate male sterility under high-temperature stress in cotton (Gossypium hirsutum). Plant J. 2017, 91, 977-994. [CrossRef] [PubMed]

32. Du, H.; Liu, H.B.; Xiong, L.Z. Endogenous auxin and jasmonic acid levels are differentially modulated by abiotic stresses in rice. Front. Plant Sci. 2013, 4, 397. [CrossRef] [PubMed]

33. Mace, E.S.; Tai, S.S.; Gilding, E.K.; Li, Y.H.; Prentis, P.J.; Bian, L.L.; Campbell, B.C.; Hu, W.S.; Innes, D.J.; Han, X.L.; et al. Whole-genome sequencing reveals untapped genetic potential in Africa's indigenous cereal crop sorghum. Nat. Commun. 2013, 4, 2320. [CrossRef] [PubMed]

34. Wang, S.K.; Bai, Y.H.; Shen, C.J.; Wu, Y.R.; Zhang, S.N.; Jiang, D.A.; Guilfoyle, T.J.; Chen, M.; Qi, Y.H. Auxin-related gene families in abiotic stress response in Sorghum bicolor. Funct. Integr. Genom. 2010, 10, 533-546. [CrossRef] [PubMed]

35. Li, S.B.; Xie, Z.Z.; Hu, C.G.; Zhang, J.Z. A Review of Auxin Response Factors (ARFs) in Plants. Front. Plant Sci. 2016, 7, 47. [CrossRef]

36. Roosjen, M.; Paque, S.; Weijers, D. Auxin Response Factors: Output control in auxin biology. J. Exp. Bot. 2018, 69, 179-188. [CrossRef] 
37. Qi, Y.H.; Wang, S.K.; Shen, C.J.; Zhang, S.N.; Chen, Y.; Xu, Y.X.; Liu, Y.; Wu, Y.R.; Jiang, D.A. OsARF12, a transcription activator on auxin response gene, regulates root elongation and affects iron accumulation in rice (Oryza sativa). New Phytol. 2012, 193, 109-120. [CrossRef]

38. Wang, S.K.; Zhang, S.N.; Sun, C.D.; Xu, Y.X.; Chen, Y.; Yu, C.L.; Qian, Q.; Jiang, D.A.; Qi, Y.H. Auxin response factor (OsARF12), a novel regulator for phosphate homeostasis in rice (Oryza sativa). New Phytol. 2014, 201, 91-103. [CrossRef]

39. Shen, C.J.; Wang, S.K.; Zhang, S.N.; Xu, Y.X.; Qian, Q.; Qi, Y.H.; Jiang, D.A. OsARF16, a transcription factor, is required for auxin and phosphate starvation response in rice (Oryza sativa L.). Plant Cell Env. 2013, 36, 607-620. [CrossRef]

40. Adamowski, M.; Friml, J. PIN-Dependent Auxin Transport: Action, Regulation, and Evolution. Plant Cell 2015, 27, 20-32. [CrossRef]

41. Bennett, T.; Hines, G.; van Rongen, M.; Waldie, T.; Sawchuk, M.G.; Scarpella, E.; Ljung, K.; Leyser, O. Connective Auxin Transport in the Shoot Facilitates Communication between Shoot Apices. PLoS Biol. 2016, 14, e1002446. [CrossRef] [PubMed]

42. Kohlen, W.; Ng, J.L.P.; Deinum, E.E.; Mathesius, U. Auxin transport, metabolism, and signalling during nodule initiation: Indeterminate and determinate nodules. J. Exp. Bot. 2018, 69, 229-244. [CrossRef] [PubMed]

43. Scarpella, E.; Marcos, D.; Friml, J.; Berleth, T. Control of leaf vascular patterning by polar auxin transport. Gene Dev. 2006, 20, 1015-1027. [CrossRef]

44. Kumar, S.; Stecher, G.; Li, M.; Knyaz, C.; Tamura, K. MEGA X: Molecular Evolutionary Genetics Analysis across Computing Platforms. Mol. Biol. Evol. 2018, 35, 1547-1549. [CrossRef] [PubMed]

45. Bailey, T.L.; Gribskov, M. Combining evidence using p-values: Application to sequence homology searches. Bioinformatics 1998, 14, 48-54. [CrossRef] [PubMed]

46. Gao, J.P.; Cao, X.L.; Shi, S.D.; Ma, Y.L.; Wang, K.; Liu, S.J.; Chen, D.; Chen, Q.; Ma, H.L. Genome-wide survey of $A u x / I A A$ gene family members in potato (Solanum tuberosum): Identification, expression analysis, and evaluation of their roles in tuber development. Biochem. Biophys. Res. Commun. 2016, 471, 320-327. [CrossRef]

47. Deng, Y.T.; Zou, W.X.; Li, G.; Zhao, J. TRANSLOCASE OF THE INNER MEMBRANE9 and 10 Are Essential for Maintaining Mitochondrial Function during Early Embryo Cell and Endosperm Free Nucleus Divisions in Arabidopsis. Plant Physiol. 2014, 166, 853-868. [CrossRef] 